\title{
Genetic associations with clozapine-induced myocarditis in patients with schizophrenia
}

Paul Lacaze 10', Kathlyn J. Ronaldson (1)', Eunice J. Zhang ${ }^{2}$, Ana Alfirevic ${ }^{2}$, Hardik Shah ${ }^{3}$, Leah Newman ${ }^{3}$, Maya Strahl ${ }^{3}$, Melissa Smith ${ }^{3}$, Chad Bousman ${ }^{4}$, Ben Francis ${ }^{5}$, Andrew P. Morris ${ }^{5,6}$, Trevor Wilson ${ }^{7}$, Fernando Rossello ${ }^{8}$, David Powell', Vivien Vasic ${ }^{7}$, Robert Sebra ${ }^{3}$, John J. McNeil ${ }^{1}$ and Munir Pirmohamed ${ }^{2}$

\begin{abstract}
Clozapine is the most effective antipsychotic drug for schizophrenia, yet it can cause life-threatening adverse drug reactions, including myocarditis. The aim of this study was to determine whether schizophrenia patients with clozapine-induced myocarditis have a genetic predisposition compared with clozapine-tolerant controls. We measured different types of genetic variation, including genome-wide single-nucleotide polymorphisms (SNPs), coding variants that alter protein expression, and variable forms of human leucocyte antigen (HLA) genes, alongside traditional clinical risk factors in 42 cases and 67 controls. We calculated a polygenic risk score (PRS) based on variation at 96 different genetic sites, to estimate the genetic liability to clozapine-induced myocarditis. Our genome-wide association analysis identified four SNPs suggestive of increased myocarditis risk $\left(P<1 \times 10^{-6}\right)$, with odds ratios ranging 5.5-13.7. The SNP with the lowest $P$ value was rs74675399 (chr19p13.3, $P=1.21 \times 10^{-7} ; \mathrm{OR}=6.36$ ), located in the GNA15 gene, previously associated with heart failure. The HLA-C*07:01 allele was identified as potentially predisposing to clozapine-induced myocarditis $(\mathrm{OR}=2.89,95 \% \mathrm{Cl}: 1.11-7.53)$, consistent with a previous report of association of the same allele with clozapine-induced agranulocytosis. Another seven HLA alleles, including HLA$B^{*} 07: 02$ ( $\left.\mathrm{OR}=0.25,95 \% \mathrm{Cl}: 0.05-1.2\right)$ were found to be putatively protective. Long-read DNA sequencing provided increased resolution of HLA typing and validated the HLA associations. The PRS explained $66 \%$ of liability ( $P$ value $=$ $\left.9.7 \times 10^{-5}\right)$. Combining clinical and genetic factors together increased the proportion of variability accounted for $\left(r^{2}\right.$ $\left.0.73, P=9.8 \times 10^{-9}\right)$. However, due to the limited sample size, individual genetic associations were not statistically significant after correction for multiple testing. We report novel candidate genetic associations with clozapine-induced myocarditis, which may have potential clinical utility, but larger cohorts are required for replication.
\end{abstract}

\section{Introduction}

Nearly 50 years after its first commercial use, clozapine continues to be the most effective antipsychotic drug for schizophrenia ${ }^{1,2}$. However, it can cause life-threatening adverse drug reactions, specifically agranulocytosis ${ }^{3}$,

\footnotetext{
Correspondence: Paul Lacaze (paul.lacaze@monash.edu)

'Department of Epidemiology and Preventive Medicine, Monash University, Melbourne, VIC, Australia

${ }^{2}$ MRC Centre for Drug Safety Science, Wolfson Centre for Personalised Medicine, University of Liverpool, The Royal Liverpool and Broadgreen University Hospitals NHS Trust, and Liverpool Health Partners, Liverpool, UK Full list of author information is available at the end of the article.

These authors contributed equally: Paul Lacaze, Kathlyn J. Ronaldson, Eunice J. Zhang
}

gastrointestinal hypomotility ${ }^{4}$ and myocarditis ${ }^{5}$. The risk of agranulocytosis is about $1 \%$ and has prompted mandatory blood monitoring protocols in most countries, and restriction to prescription as third-line therapy. Together with concerns about the risk of myocarditis and bowel obstruction, this has reduced the number of patients receiving clozapine, despite its demonstrated superior efficacy.

Myocarditis (inflammation of the heart muscle) occurs in about $3 \%$ of patients commencing clozapine, and can be fatal ${ }^{5}$. A protocol has been proposed to monitor myocarditis concurrently with monitoring agranulocytosis in the first 4 weeks following initiation of clozapine ${ }^{6}$. A

\section{(c) The Author(s) 2020}

(c) (i) Open Access This article is licensed under a Creative Commons Attribution 4.0 International License, which permits use, sharing, adaptation, distribution and reproduction c. in any medium or format, as long as you give appropriate credit to the original author(s) and the source, provide a link to the Creative Commons license, and indicate if changes were made. The images or other third party material in this article are included in the article's Creative Commons license, unless indicated otherwise in a credit line to the material. If material is not included in the article's Creative Commons license and your intended use is not permitted by statutory regulation or exceeds the permitted use, you will need to obtain permission directly from the copyright holder. To view a copy of this license, visit http://creativecommons.org/licenses/by/4.0/. 
previous case-control analysis, by our group, designed to identify clinical risk factors for clozapine-induced myocarditis, found the reaction was more common with rapid dose titration, concomitant sodium valproate and increasing age $\mathrm{e}^{7}$. Despite the investigation of a wide range of host and clinical factors including other concurrent medications, smoking status, alcohol and illicit drug use, body-mass index, and duration of psychiatric illness, the final statistical model accounted for less than $50 \%$ of the risk of myocarditis in individuals commencing clozapine. Given the wide range of factors considered, we hypothesized that genetic factors could account for a substantial proportion of the remaining risk.

Clozapine-induced myocarditis is thought to be an immune-mediated reaction, although the mechanism has not been fully elucidated. Pharmacogenetic studies of other immune-mediated adverse drug reactions have identified alleles in the human leucocyte antigen (HLA) region with sufficient predictive value for screening prior to prescribing. Two examples are Stevens-Johnson Syndrome and toxic epidermal necrolysis (SJS/TEN) with carbamazepine in those of South East Asian descent and hypersensitivity with abacavir ${ }^{8,9}$.

However, there are notable clinical differences between the adverse reactions observed with carbamazepine and abacavir, and myocarditis with clozapine. Rechallenge after abacavir hypersensitivity produces a rapid and more severe response than the initial adverse reaction ${ }^{10}$. Rechallenge to carbamazepine after SJS/TEN is strictly contra-indicated. Rechallenge with clozapine after myocarditis may lead to a recurrence; but in around $50 \%$ of cases, clozapine has been safely continued long term without discernible adverse cardiac effects ${ }^{11}$. This suggests that there may be several predisposing factors associated with clozapine-induced myocarditis. Indeed, mild cases of myocarditis have been reported, in which clozapine has been continued with doses withheld or temporarily reduced, without any long-term consequences ${ }^{12}$. This would suggest that any genetic predisposition to myocarditis is complex, and that at least for some, risk is modifiable by immunological factors akin to desensitization, or by environmental factors.

The aim of this study was to investigate the contribution of genetic factors-common and rare genetic variants together with HLA gene variants or alleles-to myocarditis risk with clozapine. To our knowledge, this is the first genetic study of clozapine-induced myocarditis.

\section{Methods}

\section{Sample description}

Cases and controls of the cohort have been described previously, along with listing of human research ethics committees from which approvals were obtained ${ }^{6,7,13}$. Briefly, cases met clinical and diagnostic criteria for myocarditis, developing within 45 days of commencing clozapine $^{13}$. Controls took clozapine for at least 45 days with no evidence of myocarditis within this period ${ }^{13}$. Those who met the criteria for case or control were invited via their case manager to provide a blood or saliva sample for genetic analysis. Samples (stored blood or tissue) from deceased individuals were obtained with the consent of the next of kin.

\section{Genome-wide association analyses}

Single-nucleotide polymorphisms (SNPs) were genotyped using the Human OmniExpressExome-8 v1.0 Bead Chip (Illumina, Inc.) in DNA samples from 42 cases, and 67 controls. Sample and SNP quality control (QC) and SNP imputation are described in the Supplementary Materials. A total of 95 samples (33 clozapine-induced myocarditis cases, 62 clozapine-tolerant controls) and 587,647 SNPs passed QC. Phasing and imputation were performed using the Haplotype Reference Consortium (HRC) panel v1.1 $2016^{14}$. Post-imputation SNP QC was performed (Supplementary Methods) and a final set of 6,822,414 bi-allelic SNPs remained and were used for association analysis.

Analysis of genotype data was conducted using an additive logistic regression model in SNPTEST v2.5 adjusting for clinical variables reaching statistical significance $(P<0.05)$ and the first four principal components from a genetic relatedness matrix to account for population stratification. The conventional genome-wide association study (GWAS) threshold of $P<5 \times 10^{-8}$ was regarded as genome-wide significant (Bonferroni-corrected for 1 million SNPs). Since this threshold is conservative for small sample sizes, SNPs with $P<1 \times 10^{-5}$ were considered suggestive of genome-wide significance.

\section{Polygenic risk score analysis}

A polygenic risk score (PRS) calculates the aggregate weighted contribution of multiple low-effect genetic loci (SNPs) on a given trait or disease outcome. We calculated a PRS for clozapine-induced myocarditis using a sub-set of SNPs meeting a more stringent QC threshold, using the PRSice-2 software ${ }^{15}$ (Supplementary Materials). We adjusted for clinical variables and the first four principal components to control for population stratification. The $R^{2}$ value was calculated to estimate the proportion of explained variance in myocarditis status.

\section{Rare coding variant analyses}

Of the SNPs assayed on the Illumina Human OmniExpressExome-8 v1.0 Bead Chip, 244,331 were coding (exonic) variants affecting protein expression. These were used to undertake a limited analysis of rare coding variants contributing to clozapine-induced myocarditis risk (see Supplementary Methods). 


\section{HLA analyses}

HLA typing was conducted using short- and long-read methods. Short-read HLA calls were made using the Omixon Target HLA Typing software (v1.8.1), following hybridization capture (SureSelect MHC, Agilent) and DNA sequencing $(2 \times 150$ bp paired end, Illumina HiSeq). Long-read, high-resolution HLA typing was performed using long-range PCR amplification and long-read single molecule real-time DNA sequencing (Pacific Biosciences RSII). For this approach, input DNA was amplified using HLA Class I and II NGSgoAmpX primers (GenDX) and purified to targeted amplicon sizes $(\sim 3-4 \mathrm{~kb}$ for HLA-A, $-B$ and $-C)$. After PCR products were normalized and pooled, they were subject to size selection, template preparation, barcode multiplexing (eight libraries per pool) and SMRT sequencing using magnetic bead selection protocol. Long-read data were processed using the Long Amplicon Analysis pipeline v2 (Pacific Biosciences). FASTQ files were imported into the NGSengine v2.7.0 software (GenDX) for HLA allele calling, SNP identification and individual base calling.

Short-read DNA sequencing enabled HLA typing at standard (4-digit) resolution. This detected 29 HLA loci and 4 non-HLA loci across the major histocompatibility complex (MHC) (Table S1). Loci with call rates $<98 \%$ were excluded $(n=7)$. Of the remaining 26 loci, 313 alleles were detected (Table S2). Monomorphic alleles $(n=$ $25)$ and alleles with counts of $<1 \quad(n=86)$ were excluded. To minimize co-linearity, only SNPs with correlation coefficient $r^{2}<0.8$ were included. A final number of 127 HLA and 32 non-HLA alleles in 33 cases and 62 controls were included for downstream analyses. HLA alleles were grouped into supertypes (Table S3) according to published classifications ${ }^{16,17}$. The frequency of HLA haplotype was explored in publicly available Caucasoid populations using the 'haplotype frequency search' option on the allele frequency net database.

To evaluate the effect of each individual HLA allele, HLA supertype and HLA haplotype on clozapine-induced myocarditis, logistic regression analysis was undertaken using PLINK software ${ }^{18}$, assuming a dominant mode of inheritance, with adjustment for clinical variables with $P<$ 0.05 . Correction for multiple testing was undertaken using Benjamini-Hochberg false discovery rate $(F D R)^{19}$ in the genetics package of $\mathrm{R}$ (v3.3.1).

Long-read HLA sequencing was performed to improve accuracy and resolution of the short-read HLA sequencing results, to resolve ambiguities and to generate highresolution (eight-digit) HLA calls. Long-read sequencing detected 11 HLA loci (HLA-A, B, C, DRB1, DRB3, DRB4, $D R B 5, D Q A 1, D Q B 1, D P A 1$, and DPB1). HLA loci with $>90 \%$ call rate (HLA-A, B, and C) were included in the concordance check with short-read HLA sequencing data.

\section{Clinical variables and statistical analyses}

Statistical associations of clinical variables (sex, smoking, alcohol abuse, illicit drug use, valproate use, chronic disease, age, BMI, and cumulative clozapine dose) with clozapineinduced myocarditis were evaluated using either the Pearson's chi-square test or ANOVA (see Supplementary Methods). Clinical covariates found to be significantly associated with clozapine-induced myocarditis were combined with genetic factors using regression models, to calculate the proportion of variability accounted for.

\section{Results}

Table 1 provides a summary of the case-control cohort, clinical variables and genomic measures, as well as numbers included in each analysis. Exposure to potentially interacting medication via cytochrome P450 metabolism was minimal among cases and controls $(n=0-4$; Table S4).

Table 1 Cohort description and numbers.

\begin{tabular}{|c|c|c|}
\hline & Cases & Controls \\
\hline Number & 42 & 67 \\
\hline \multicolumn{3}{|l|}{ Sample type } \\
\hline Fresh blood/saliva/stored blood/tissue & $20 / 11 / 6 / 5$ & $61 / 6 / 0 / 0$ \\
\hline \multicolumn{3}{|l|}{ Age at clozapine initiation } \\
\hline Mean age (years) $\pm S D$ & $37 \pm 12$ & $35 \pm 11$ \\
\hline \multicolumn{3}{|l|}{ Reported ethnicity } \\
\hline European/other/unknown & $36 / 2 / 4$ & $57 / 7 / 3$ \\
\hline Outcome fatal & $24 \%(10 / 42)$ & - \\
\hline Sex (\% male) & $71 \%(30 / 42)$ & $81 \%(54 / 67)$ \\
\hline \multicolumn{3}{|l|}{ Clinical factors } \\
\hline Smoking-Y/N/Unknown & $29 / 7 / 6$ & $49 / 18 / 0$ \\
\hline Alcohol abuse-Y/N/Unknown & $10 / 25 / 7$ & $9 / 46 / 12$ \\
\hline Illicit drug use-Y/N/Unknown & $8 / 27 / 7$ & $12 / 46 / 9$ \\
\hline Valproate use-Y/N/Unknown & $12 / 26 / 4$ & $9 / 58 / 0$ \\
\hline $\mathrm{BMI} \mathrm{kg} / \mathrm{m}^{2} \pm \mathrm{SD}$ & $\begin{array}{l}31.7 \pm 6.8 \\
(n=30)\end{array}$ & $\begin{array}{l}27.8 \pm 6.5 \\
(n=54)\end{array}$ \\
\hline \multicolumn{3}{|l|}{ Cumulative clozapine dose-days 1-9 } \\
\hline Mean $(\mathrm{mg}) \pm \mathrm{SD}$ & $\begin{array}{l}751 \pm 222 \\
(n=35)\end{array}$ & $\begin{array}{l}691 \pm 289 \\
(n=62)\end{array}$ \\
\hline \multicolumn{3}{|l|}{ Genetic analyses-QC pass rate ${ }^{a}$} \\
\hline 1. Genome-wide SNP association & $79 \%(33 / 42)$ & $93 \%(62 / 67)$ \\
\hline 2. Rare variants/gene-level analysis & $71 \%(30 / 42)$ & $81 \%(54 / 67)$ \\
\hline 3. Targeted short-read HLA typing & $93 \%(33 / 42)$ & $96 \%(62 / 67)$ \\
\hline 4. Long-read HLA typing/phasing & $71 \%(30 / 42)$ & $90 \%(60 / 67)$ \\
\hline
\end{tabular}

${ }^{\mathrm{a}}$ For reasons for exclusion, see Supplementary methods. 


\section{Genome-wide single-nucleotide polymorphism (SNP) association}

Our analysis found no SNPs reaching genome-wide significance between cases and controls (Fig. 1). However, SNPs with suggestive genome-wide significance were observed in several regions. In particular, there were four SNPs located on chromosomes 2p21, 6p12.2, 8p21.2, and 19p13.3 (Table 2, Fig. 2) with $P<1 \times 10^{-6}$. The frequencies of these four variants were at least $20 \%$ higher in cases compared to controls.

The SNP with the lowest $P$ value was rs74675399 located on chromosome $19 \mathrm{p} 13.3 \quad\left(P=1.21 \times 10^{-7} ; \quad\right.$ OR $=6.36$; Fig. 2a), within intron 2 of the GNA15 gene. This gene encodes the G-protein subunit alpha-15 and has previously been reported in genome-wide association with heart failure $^{20}$. The second lowest $P$ value was associated with an intergenic SNP, rs117188076 $\left(P=2.98 \times 10^{-7}\right.$; OR = 13.74; Fig. 2b), located on chromosome $8 \mathrm{p} 21.2$. This variant is located $\sim 31 \mathrm{~kb}$ from the $3^{\prime}$ end of the disintegrin and metalloproteinase domain-containing protein 7 gene (ADAM7). The third was also an intergenic SNP, rs2959223 $\left(P=3.5 \times 10^{-7} ;\right.$ OR $=5.55 ;$ Fig. $\left.2 \mathrm{c}\right)$, positioned $\sim 8.6 \mathrm{~kb}$ from the $3^{\prime}$ end of transcription factor SIX2 that encodes for homeobox protein SIX2 on chromosome 2p21. The fourth was an intronic EFHC1 SNP, rs9463787 $\left(P=8.08 \times 10^{-7}\right.$; $\mathrm{OR}=7.67$; Fig. 2d) on chromosome 6p12.2. EFHC1 encodes the EF-hand-containing calcium binding protein, which plays a role in calcium homoeostasis.

\section{Polygenic risk score (PRS)}

PRS was calculated using SNP effect sizes (odds ratio) from the GWAS analysis. Ninety-six SNPs with $P<1 \times 10^{-5}$ explained $66 \%$ of variance in clozapine-induced myocarditis $\left(\right.$ PRS $P$ value $\left.=9.7 \times 10^{-5}\right)($ Fig. 3$)$.

\section{Exonic variants/gene-based association}

A total of 226,140 exonic variants in protein-coding gene regions genotyped across 30 clozapine-induced myocarditis cases, and 54 clozapine-tolerant controls passed quality control for gene-based analysis. No significant association was found between the exonic markers and clozapine-induced myocarditis with regard to detectable disruption in gene function (Fig. S1).

\section{HLA association}

The short-read DNA sequencing enables HLA typing to two-digit resolution. This identified nine HLA gene variant (allele) associations with clozapine-induced myocarditis (Table 3), prior to false discovery rate (FDR) correction to address type I errors. HLA class I allele HLA-C*07:01 (OR = 2.89, 95\% CI: 1.11-7.53; $P=0.03)$ and HLA class II allele $H L A-D M B * 01: 01(\mathrm{OR}=3.22,95 \%$ CI: $1.158 .97 ; P=0.026)$ were associated with increased odds for myocarditis.

The other seven HLA alleles were all associated with decreased frequency of myocarditis (ORs $<1)$. Two of these were HLA class I alleles (HLA-B*07:02 and $H L A$ $C^{* 07: 02)}$ and four were HLA class II alleles (HLA$D Q A 1 * 01: 02, H L A-D Q B 1 * 06: 02, H L A-D R A * 01: 01$ and $H L A-D R B 1 * 15: 01)$. One was a non-HLA allele, $M I C B^{*} 004: 01$. In addition to allele associations, one HLA supertype, DR1, exhibited a significant association with decreased frequency of clozapine-induced myocarditis $(\mathrm{OR}<1$; Table 3$)$. However, none of the allelic and supertype associations remained significant after FDR.

Using the allele frequency net database ${ }^{21}, H L A-B * 07: 02$, $D Q B 1 * 06: 02$ and $D R B 1 * 15: 01$ were found to be in the same haplotype group. Among 44 records focusing on the
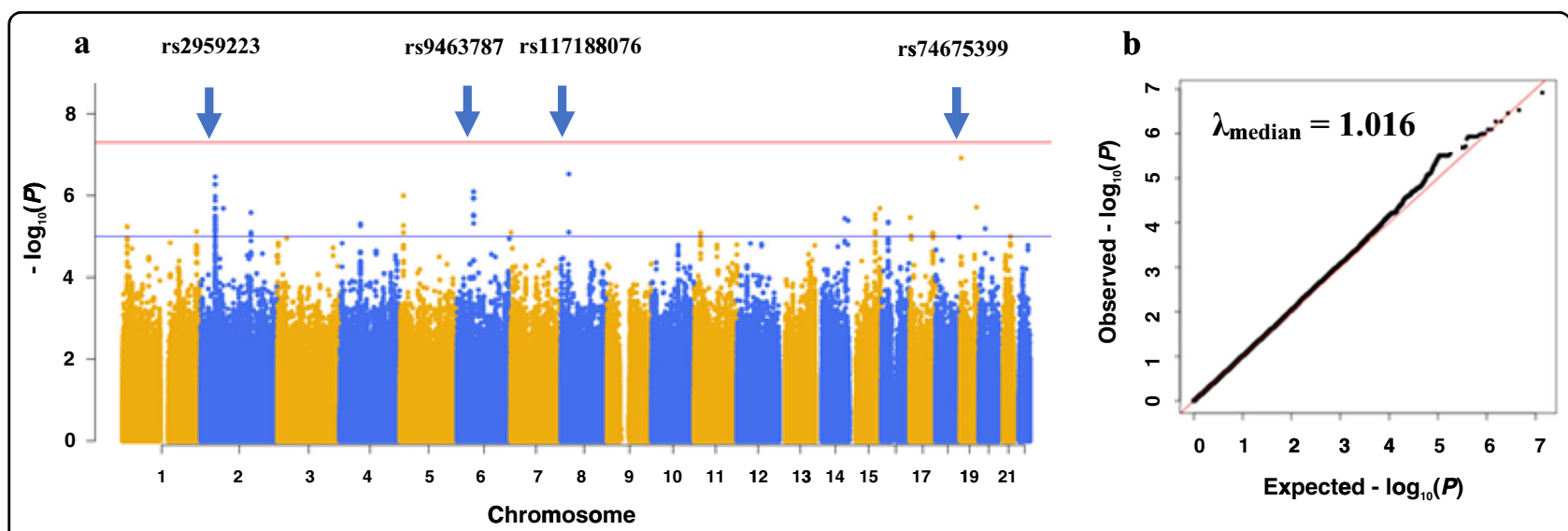

Fig. 1 Association of genome-wide genetic variants in clozapine-induced myocarditis cases compared with clozapine-tolerant controls. The first four principal components and valproate use were included as covariates. a Manhattan plot showing individual $-\log _{10} P$ values plotted against their genomic position by chromosome. The red line represents the threshold of genome-wide significance $\left(P=5 \times 10^{-8}\right)$. The blue line marks the threshold for suggestive association $\left(P=1 \times 10^{-5}\right)$. Blue arrows indicate SNPs with $P<1 \times 10^{-6}$. b Quantile-quantile (Q-Q) plot of data shown in Manhattan plot. 


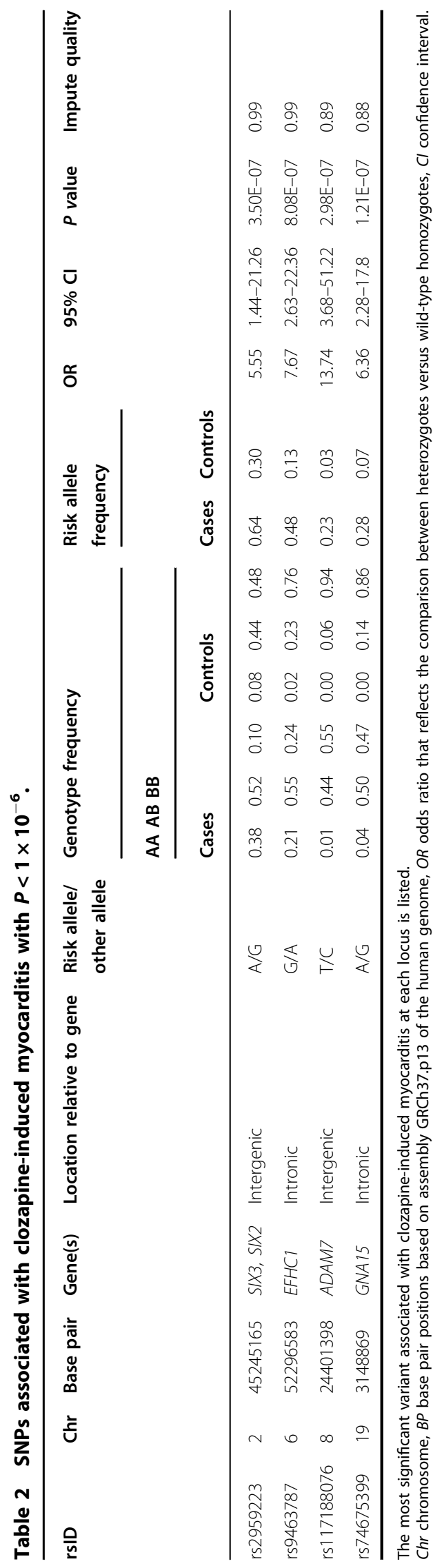

Caucasoid ethnic origin across seven populations, consisting of a total of 1,250,628 individuals, the estimated occurrence of the HLA-B*07:02, DQB1*06:02 and $D R B 1 * 15: 01$ haplotypes ranges between 0.01 and $11 \%$. In our cohort of clozapine-induced myocarditis cases and controls, the carrier frequency of these three alleles together was $9.1 \%$ and $22.6 \%$, respectively $(\mathrm{OR}=0.25$, 95\% CI: $0.05-1.2 ; P=0.083)$. This suggests that differences observed in $H L A-B * 07: 02, D Q B 1^{*} 06: 02$ and $D R B 1 * 15: 01$ frequencies between cases and controls in our study are not likely to be due to confounding differences in population structure.

\section{Long-read HLA typing}

Long-read DNA sequencing can span highly variable HLA gene regions, to enable typing at higher resolution, to four-digits. Long-read sequencing was used to validate and further resolve HLA calls made by short-read sequencing in clozapine-induced myocarditis cases and controls. Among the 96 samples that underwent longread HLA DNA sequencing, 90 had short-read HLA sequencing data available, of which 86 had successful long-read HLA-B PCR results (Table S5).

All $H L A-B * 07: 02$ calls based on short-read data were identified as $H L A-B^{*} 07: 02: 01: 01$ by long-read analysis. Eighty samples had successful long-read HLA-C results. All short-read calls for HLA-C*07:01 and *07:02 were concordant with long-read typing. Carriers of the loci previously associated with clozapine-induced agranulocytosis ${ }^{22}$, HLA-C*07:01 $(n=31)$, were identified as having HLA$C * 07: 01: 01: 01 \quad(n=30)$ and HLA-C*07:01:01:04 $(n=1$; control). The $H L A-C * 07: 02$ carriers $(n=31)$ were identified as having $H L A-C^{*} 07: 02: 01: 03 \quad(n=30)$ and HLA$C$ 07:02:01:01 ( $n=1$; case). Discordance between HLA typing calls from short- and long-read DNA sequencing was observed across five samples (Table S6), but this did not alter the statistical findings of the short-read HLA analysis.

\section{Regression analysis for myocarditis with clozapine}

The univariate associations of clinical variables with clozapine-induced myocarditis are summarized in Table S7. We found that the concomitant use of valproate with clozapine contributes $6.8 \%$ to the risk of myocarditis $(\mathrm{OR}=$ 3.1, 95\% CI: $1.1-8.9 ; P=0.03$ ) (Table 4). The addition of HLA and non-HLA alleles increased the $r^{2}$ by approximately $24 \%$. When the top four GWAS SNPs were included, a further $42 \%$ increase in $r^{2}$ was observed. When clinical and genetic factors were combined, they explained a higher proportion of risk (72.6\%) compared to clinical factors alone.

\section{Discussion}

This study provides the first evidence, to our knowledge, that genetic factors contribute to the risk of myocarditis 


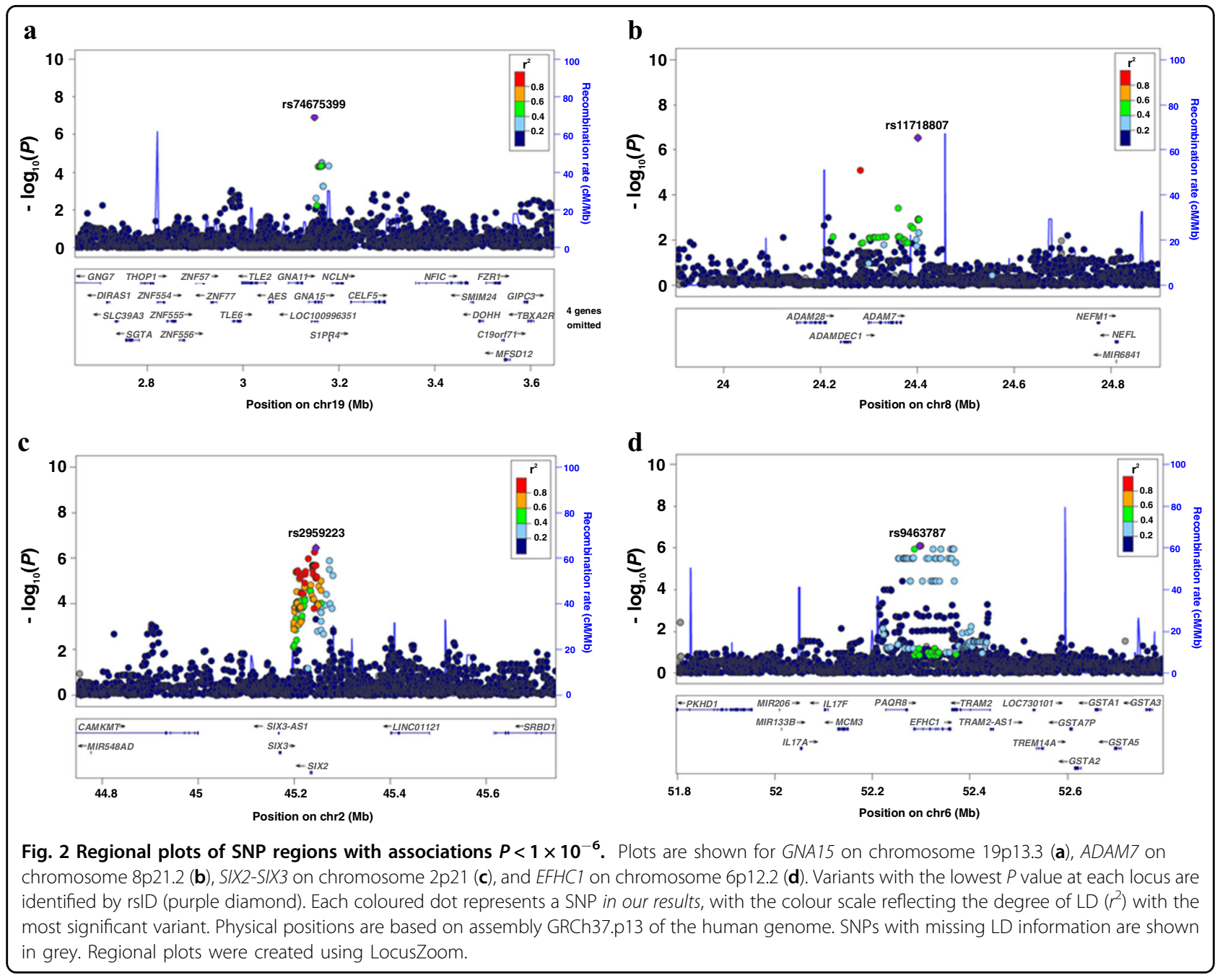

with clozapine. Although the genetic associations identified did not reach the conventional statistical threshold used in human genetics, and require replication in larger studies, they provide a novel set of candidate genetic loci for this serious adverse drug reaction, and may be of potential clinical utility.

A strength of our study is the comprehensive genetic analyses undertaken using a range of technologies used to measure different types of genetic variation, including genome-wide SNP variation, HLA variation and polygenic risk. Our results have prompted collaborative efforts to increase sample sizes to improve statistical power to detect difference, through an international clozapineinduced myocarditis consortium ${ }^{23}$.

Several previous genetic studies have been conducted on clozapine-induced agranulocytosis ${ }^{24}$. These suggest agranulocytosis with clozapine may be a heritable trait, with a complex genetic architecture where numerous genetic loci contribute, including the HLA genes ${ }^{24}$, in combination with clinical factors. Further, a recent genome-wide association study (GWAS) of neutropenia in patients taking clozapine identified an association with $H L A-D Q B 1^{25}$. Together, these previous studies suggest a small, but significant role of genetic factors contributing to the risk of clozapine-induced agranulocytosis and neutropenia, but no single polymorphism with high predictive value or current clinical utility. Similarly, the results of our study suggest that the risk of myocarditis with clozapine is underpinned by a complex genetic architecture.

Following either myocarditis or agranulocytosis/neutropenia, the outcome of rechallenge with clozapine may be favourable ${ }^{11,26}$. In addition, clozapine has been safely continued in mild cases of myocarditis ${ }^{12}$. These observations suggest that the pathogenesis may not always be a typical immune-mediated reaction, but one where a complex combination of genetic and clinical factors lead to predisposition, and modification of one or more of these, or a process akin to desensitization, may modulate risk.

From a biological standpoint, we identified genetic risk loci that provide clues to the underlying aetiology of myocarditis with clozapine. These loci include four novel 


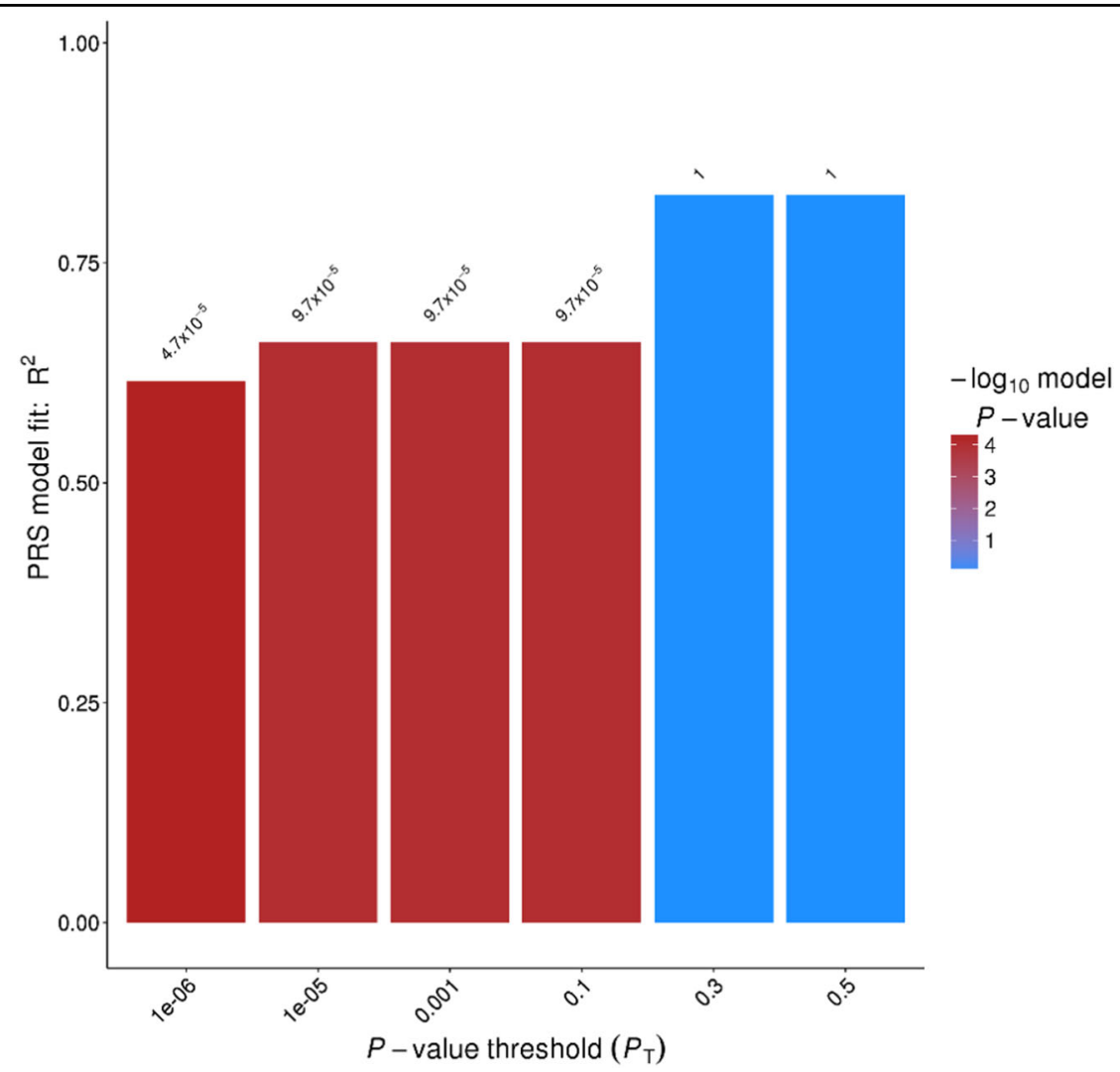

Fig. 3 Polygenic risk score analysis using GWAS genetic variants. Risk scores of pruned SNPs at each predetermined significance threshold were calculated using aggregated sum of weighted risk allele effect sizes. The proportion of variance $\left(R^{2}\right)$ explained by the aggregate risk score is shown on the $y$-axis.

SNPs that approached genome-wide significance, even in our small sample size. While we found no associations above the standard statistical threshold for genome-wide SNP significance $\left(P<5 \times 10^{-8}\right)$, there are instances of SNPs discovered below this threshold being biologically meaningful, and reaching genome-wide significance subsequently, after larger sample sizes are available ${ }^{27}$. We therefore find it reasonable to interpret the biological consequences and potential clinical utility of the genetic variation we have identified, albeit with due caution.

The locus with the lowest associated $P$ value was located within intron 2 of the GNA15 gene and is associated with six-fold increase in susceptibility to clozapine-induced myocarditis. This locus has previously been associated with risk of incident heart failure in adults of European ancestry $^{20}$. The GNA15 gene encodes a Ga/q protein that plays a role in a variety of signalling pathways important for heart function ${ }^{28}$. GNA15 sits in a genomic region (19p13.3) where microdeletions have been linked to congenital heart disease ${ }^{29}$. The implicated variant (rs74675399) sits 132 base pairs upstream from an enhancer region and 1.7 kilobases downstream of a promoter flanking region, suggesting it may act as a proxy for functional genomic elements. Further, clozapine has high binding affinities to receptors that GNA15 is functionally coupled to, such as 5-HT2 serotonergic, muscarinic (M1, M3, M4), alpha-1 adrenergic, and histamine H1 receptors ${ }^{30}$. GNA15 is also involved in lymphocyte and neutrophil activation after pro-inflammatory cytokine treatment $^{31,32}$. Even with the caveats of limited sample size and conservative type 1 error threshold, this provides a promising candidate locus with biological plausibility, related to the clinical phenotype observed.

With regards to HLA associations, statistical thresholds and FDR correction also apply to reduce the possibility of type I error. We found potential novel HLA associations with clozapine-induced myocarditis, including the HLA class I allele $H L A-C * 07: 01$ (OR $=2.89,95 \%$ CI: $1.11-7.53$; $P=0.03)$, previously associated with clozapine-induced agranulocytosis $^{22}$. This result suggested an overlapping role of $H L A-C * 07: 01$ in the risk of different clinically significant clozapine-induced adverse drug reactions. Interestingly, $H L A-C^{*} 07: 01$ has also been associated with the response to peg-interferon alfa- $2 \mathrm{~b}$ and ribavirin therapy in 
Table 3 Associations of HLA and non-HLA alleles with clozapine-induced myocarditis showing $P<0.05$ prior to FDR. Valproate use was included in the analyses as covariate.

\begin{tabular}{|c|c|c|c|c|c|c|}
\hline \multirow[t]{2}{*}{ Allele/supertype } & \multicolumn{2}{|l|}{ Cases } & \multicolumn{2}{|c|}{ Controls } & \multirow[t]{2}{*}{ OR $(95 \% \mathrm{Cl})$} & \multirow[t]{2}{*}{$P^{\mathrm{a}}$} \\
\hline & Carrier & Non-carrier & Carrier & Non-carrier & & \\
\hline \multicolumn{7}{|l|}{ HLA Class I } \\
\hline HLA-B*07:02 & 0.10 & 0.90 & 0.32 & 0.68 & $0.26(0.07-0.98)$ & 0.047 \\
\hline HLA-C*07:01 & 0.55 & 0.45 & 0.34 & 0.66 & $2.89(1.11-7.53)$ & $0.030^{\mathrm{a}}$ \\
\hline HLA-C*07:02 & 0.10 & 0.90 & 0.34 & 0.66 & $0.25(0.07-0.92)$ & 0.037 \\
\hline \multicolumn{7}{|l|}{ HLA Class II } \\
\hline HLA-DMB*01:01 & 0.76 & 0.24 & 0.52 & 0.48 & $3.22(1.15-8.97)$ & $0.026^{a}$ \\
\hline HLA-DQA 1*01:02 & 0.17 & 0.83 & 0.45 & 0.55 & $0.23(0.074-0.71)$ & 0.011 \\
\hline HLA-DQB1*06:02 & 0.10 & 0.90 & 0.35 & 0.65 & $0.17(0.045-0.69)$ & 0.012 \\
\hline HLA-DRA*01:01 & 0.34 & 0.66 & 0.68 & 0.32 & $0.27(0.11-0.70)$ & 0.007 \\
\hline HLA-DRB1*15:01 & 0.10 & 0.90 & 0.35 & 0.65 & $0.17(0.045-0.69)$ & 0.012 \\
\hline \multicolumn{7}{|l|}{ Non-HLA } \\
\hline MICB*004:01 & 0.21 & 0.79 & 0.48 & 0.52 & $0.27(0.09-0.77)$ & 0.015 \\
\hline \multicolumn{7}{|l|}{ HLA supertype } \\
\hline HLA Class \| DR $1^{\mathrm{b}}$ & 0.17 & 0.83 & 0.39 & 0.61 & $0.28(0.09-0.89)$ & 0.031 \\
\hline
\end{tabular}

${ }^{a} P$ values did not remain significant after FDR.

${ }^{b}$ The HLA-DRB1 supertype consists of DRB1*15:01, ${ }^{*} 15: 02,{ }^{*} 15: 03,{ }^{*} 15: 04,{ }^{*} 16: 01$, and ${ }^{*} 16: 02$.

Lines in bold text indicate increased risk of myocarditis.

Table 4 Contribution of clinical and genetic variables to clozapine-induced myocarditis.

\begin{tabular}{|c|c|c|}
\hline Variable & Nagelkerke's $r^{2}$ & $P$ \\
\hline Clinical only (valproate use) & 0.068 & 0.029 \\
\hline Clinical + HLA & 0.307 & 0.021 \\
\hline Clinical + HLA + GWAS ${ }^{b}$ & 0.726 & $9.838 \mathrm{E}-09$ \\
\hline
\end{tabular}

Included HLA-B*07:02, HLA-C*07:01, HLA-C*07:02, HLA-DMB*01:01, HLADQA $1 * 01: 02, \quad$ HLADQB1*06:02, HLA-DRA*01:01， HLA-DRB1*15:01, and MICB*004:01. HLA-DRB1*15:01 was excluded from the model due to redundancy.

bIncluded GWAS top four associations rs2959223, rs9463787, rs117188076, rs74675399. Bold text indicates statistical signifcance $P<0.001$.

hepatitis $C^{33}$. When we typed $C^{*} 07: 01$ patients using highresolution, long-read HLA sequencing, we found 30/31 carriers shared the HLA-C*07:01:01:01 sub-type.

We identified seven HLA alleles associated with the decreased frequency (risk) of clozapine-induced myocarditis (ORs $<1$ ), including the $H L A-B^{*} 07: 02$ allele $(\mathrm{OR}=$ 0.26, 95\% CI: 0.07-0.98; $P=0.047)$. HLA $B * 07: 02$ has been previously associated, by our group, with putative protective function against carbamazepine hypersensitivity (Stevens-Johnson syndrome) in Caucasian patients ${ }^{34}$. High-resolution typing showed that all $H L A-B * 07: 02$ carriers were $H L A-B * 07: 02: 01: 01$ sub-types. Of all patients with HLA-B calls by long-read sequencing, the HLA$B * 07: 02: 01: 01$ allele was present in 18 of 55 controls (33\%) but only 4 of $33(12 \%)$ cases $(P=0.0375)$.

Together, our results indicate contributions from multiple genetic loci and HLA alleles to the risk of myocarditis with clozapine, suggesting a complex and polygenic architecture ${ }^{35}$. To explore this further, we generated a polygenic risk score using 96 SNPs $(P<1 \times$ $\left.10^{-5}\right)$. This explained $66 \%$ of the liability to clozapineinduced myocarditis $\left(P=9.7 \times 10^{-5}\right)$. This was a high proportion of variability for an exploratory PRS model, and provides encouraging evidence that use of genetic information may benefit risk management of clozapineinduced myocarditis.

In our previous case-control study examining clinical risk factors for clozapine-induced myocarditis (105 cases, 296 controls), we found that risk of myocarditis in individuals commencing clozapine was increased by rapid dose titration, greater patient age and concomitant sodium valproate use ${ }^{7}$. In the present study, we confirmed the association with valproate use $\left(r^{2} 0.07, P=0.03\right)$, and found that combining clinical and genetic factors together substantially increased the proportion of variability accounted for $\left(r^{2} 0.73, P=9.8 \times 10^{-9}\right)$. This provided further evidence that the addition of genetic information to clinical risk models for clozapine-induced myocarditis 
may be of potential future clinical utility in risk management and prevention. However, the genetic associations identified require validation in larger replication studies.

There could also be yet undiscovered genetic factors contributing to myocarditis risk not detected by our current methods. These include rare, high-effect size genetic variants detectable by whole-genome or wholeexome sequencing, large structural variants, or functional haplotypes in genes relevant to clozapine metabolism (e.g. CYP1A2).

\section{Limitations}

The small sample size of the cohort is the most notable limitation of this study. We spent a number of years and considerable resources recruiting even this small number of patients, encountering a range of challenges. These included the low population frequency of schizophrenia (prevalence $\sim 1 / 100$ ), the restrictions on clozapine prescribing, the reluctance of psychiatrists to prescribe clozapine due to its adverse effects, and the rarity of the drug reaction amongst patients commencing clozapine (estimated 3/100 patients treated). The stringency of clinical criteria used to define cases also contributed ${ }^{13}$. The sample set was further narrowed by the mental illness of some potential participants who were not able to be contacted about contributing a sample, or had cognitive barriers to participation. Poor DNA quality also contributed to failures for some of the genomic assays used, further reducing statistical power. In addition, with regards to measuring rare exonic variants, our analysis was restricted to only a small sub-set of coding variants included on the genotyping array used.

\section{Conclusion}

We report possible novel genetic associations with clozapine-induced myocarditis, some of which overlap with related adverse drug reactions. Our results provide candidate mechanisms by which clozapine could induce myocardial inflammation and damage, and identify candidate genetic loci that may, in aggregate, be of future clinical utility for preventing a serious and sometimes fatal adverse drug reaction. Our results warrant further investigations for this important clinical phenotype, and validation in larger studies.

\section{Acknowledgements}

C.B. is supported by the Cumming School of Medicine and Alberta Children's Hospital Foundation. M.P. is supported by the MRC Centre for Drug Safety Science, and is an Emeritus NIHR Senior Investigator.

\section{Author details}

'Department of Epidemiology and Preventive Medicine, Monash University, Melbourne, VIC, Australia. ${ }^{2}$ MRC Centre for Drug Safety Science, Wolfson Centre for Personalised Medicine, University of Liverpool, The Royal Liverpool and Broadgreen University Hospitals NHS Trust, and Liverpool Health Partners,
Liverpool, UK. ${ }^{3}$ Department of Genetics and Genomic Sciences, Icahn Institute for Genomics and Multiscale Biology, Icahn School of Medicine at Mount Sinai, New York, NY, USA. ${ }^{4}$ Departments of Medical Genetics, Psychiatry, and Physiology \& Pharmacology, University of Calgary, Calgary, AB, Canada. ${ }^{5}$ Department of Biostatistics, University of Liverpool, Liverpool, UK. ${ }^{6}$ Division of Musculoskeletal and Dermatological Sciences, University of Manchester, Manchester, UK. ${ }^{7}$ Medical Genomics Facility, Hudson Institute of Medical Research, Melbourne, VIC, Australia. ${ }^{8}$ University of Melbourne Centre for Cancer Research, University of Melbourne, Melbourne, VIC, Australia. ${ }^{9}$ Bioinformatics Platform, Monash Biomedicine Discovery Institute, Monash University, Melbourne, VIC, Australia

\section{Conflict of interest}

The authors declare that they have no conflict of interest.

\section{Publisher's note}

Springer Nature remains neutral with regard to jurisdictional claims in published maps and institutional affiliations.

Supplementary Information accompanies this paper at (https://doi.org/ 10.1038/s41398-020-0722-0).

Received: 3 June 2019 Revised: 9 December 2019 Accepted: 30 December 2019

Published online: 27 January 2020

\section{References}

1. Stroup, T. S., Gerhard, T., Crystal, S., Huang, C. \& Olfson, M. Comparative effectiveness of clozapine and standard antipsychotic treatment in adults with schizophrenia. Am. J. Psychiatry 173, 166-173 (2016).

2. Leucht, S. et al. Comparative efficacy and tolerability of 15 antipsychotic drugs in schizophrenia: a multiple-treatments meta-analysis. Lancet 382, 951-962 (2013).

3. Krupp, P. \& Barnes, P. Clozapine-associated agranulocytosis: risk and aetiology. Br. J. Psychiatry Suppl. 17, 38-40 (1992).

4. Every-Palmer, S., Inns, S. J., Grant, E. \& Ellis, P. M. Effects of clozapine on the gut: cross-sectional study of delayed gastric emptying and small and large intestinal dysmotility. CNS Drugs 33, 81-91 (2019).

5. Ronaldson, K. J., Fitzgerald, P. B. \& McNeil, J. J. Clozapine-induced myocarditis, a widely overlooked adverse reaction. Acta Psychiatr. Scand. 132, 231-240 (2015).

6. Ronaldson, K. J., Fitzgerald, P. B., Taylor, A. J., Topliss, D. J. \& McNeil, J. J. A new monitoring protocol for clozapine-induced myocarditis based on an analysis of 75 cases and 94 controls. Aust. N. Z. J. Psychiatry 45, 458-465 (2011).

7. Ronaldson, K. J. et al. Rapid clozapine dose titration and concomitant sodium valproate increase the risk of myocarditis with clozapine: a case-control study. Schizophr. Res. 141, 173-178 (2012).

8. Chung, W. H. et al. Medical genetics: a marker for Stevens-Johnson syndrome. Nature 428, 486 (2004).

9. Mallal, S. et al. HLA-B*5701 screening for hypersensitivity to abacavir. N. Engl. J. Med. 358, 568-579 (2008).

10. Escaut, L., Liotier, J. Y., Albengres, E., Cheminot, N. \& Vittecoq, D. Abacavir rechallenge has to be avoided in case of hypersensitivity reaction. AIDS 13, 1419-1420 (1999).

11. Ronaldson, K. J., Fitzgerald, P. B., Taylor, A. J. \& McNeil, J. J. Observations from 8 cases of clozapine rechallenge after development of myocarditis. J. Clin. Psychiatry 73, 252-254 (2012).

12. Ronaldson, K. J., Fitzgerald, P. B., Taylor, A. J. \& McNeil, J. J. Continuation of clozapine following mild myocarditis. Aust. N. Z. J. Psychiatry 46, 910-911 (2012).

13. Ronaldson, K. J. et al. Diagnostic characteristics of clozapine-induced myocarditis identified by an analysis of 38 cases and 47 controls. J. Clin. Psychiatry 71, 976-981 (2010).

14. Das, S. et al. Next-generation genotype imputation service and methods. Nat. Genet. 48, 1284-1287 (2016).

15. Euesden, J., Lewis, C. M. \& O'Reilly, P. F. PRSice: Polygenic Risk Score software. Bioinformatics 31, 1466-1468 (2015). 
16. Doytchinova, I. A. \& Flower, D. R. In silico identification of supertypes for class II MHCs. J. Immunol. 174, 7085-7095 (2005).

17. Sidney, J., Peters, B., Frahm, N., Brander, C. \& Sette, A. HLA class I supertypes: a revised and updated classification. BMC Immunol. 9, 1-15 (2008).

18. Purcell, S. et al. PLINK: a tool set for whole-genome association and population-based linkage analyses. Am. J. Hum. Genet. 81, 559-575 (2007).

19. Benjamini, Y., Drai, D., Elmer, G., Kafkafi, N. \& Golani, I. Controlling the false discovery rate in behavior genetics research. Behav. Brain Res. 125, 279-284 (2001).

20. Smith, N. L. et al. Association of genome-wide variation with the risk of incident heart failure in adults of European and African ancestry: a prospective meta-analysis from the cohorts for heart and aging research in genomic epidemiology (CHARGE) consortium. Circulation Cardiovascular Genet. 3, 256-266 (2010).

21. González-Galarza, F. F. et al. Allele frequency net 2015 update: new features for HLA epitopes, KIR and disease and HLA adverse drug reaction associations. Nucleic Acids Res. 43, D784-D788 (2015).

22. Dettling, M., Schaub, R. T., Mueller-Oerlinghausen, B., Roots, I. \& Cascorbi, I. Further evidence of human leukocyte antigen-encoded susceptibility to clozapine-induced agranulocytosis independent of ancestry. Pharmacogenetics 11, 135-141 (2001).

23. Bousman, C. et al. The Pharmacogenomics of Clozapine-Induced myocarditis (PROCLAIM) Consortium. ACNP 57(th) Annual Meeting: Poster Session III. Neuropsychopharmacology 2018, S97-S98 (2018).

24. de With, S. A. J., Pulit, S. L., Staal, W. G., Kahn, R. S. \& Ophoff, R. A. More than 25 years of genetic studies of clozapine-induced agranulocytosis. Pharmacogenomics J. 17, 304-311 (2017).

25. Legge, S. E. et al. Genome-wide common and rare variant analysis provides novel insights into clozapine-associated neutropenia. Mol. Psychiatry 22, 1509 (2017).
26. Manu, P., Sarpal, D., Muir, O., Kane, J. M. \& Correll, C. U. When can patients with potentially life-threatening adverse effects be re-challenged with clozapine? A systematic review of the published literature. Schizophrenia Res. 134, 180-186 (2012).

27. Kuo, H. M. K. Multiple testing in the context of gene discovery in sickle cell disease using genome-wide association studies. Genom. Insights 10, 1178631017721178 (2017).

28. Della Rocca, G. J. et al. Ras-dependent mitogen-activated protein kinase activation by $\mathrm{G}$ protein-coupled receptors. Convergence of Gi- and Gqmediated pathways on calcium/calmodulin, Pyk2, and Src kinase. J. Biol. Chem. 272, 19125-19132 (1997).

29. Peddibhotla, S. et al. Expanding the genotype-phenotype correlation in subtelomeric 19p13.3 microdeletions using high resolution clinical chromosomal microarray analysis. Am. J. Med. Genet. A 161A, 2953-2963 (2013).

30. Qin, K., Dong, C., Wu, G. \& Lambert, N. A. Inactive-state preassembly of G (q)-coupled receptors and $\mathrm{G}(\mathrm{q})$ heterotrimers. Nat. Chem. Biol. 7, 740-747 (2011).

31. Giannone, F. et al. The puzzling uniqueness of the heterotrimeric G15 protein and its potential beyond hematopoiesis. J. Mol. Endocrinol. 44, 259-269 (2010)

32. French, S. W. et al. The role of the IL-8 signaling pathway in the infiltration of granulocytes into the livers of patients with alcoholic hepatitis. Exp. Mol. Pathol. 103, 137-140 (2017).

33. Suppiah, V. et al. IL28B, HLA-C, and KIR variants additively predict response to therapy in chronic hepatitis $C$ virus infection in a European Cohort: a crosssectional study. PLoS Med. 8, e1001092 (2011).

34. Alfirevic, A. et al. HLA-B locus in Caucasian patients with carbamazepine hypersensitivity. Pharmacogenomics 7, 813-818 (2006).

35. Osanlou, O., Pirmohamed, M. \& Daly, A. K. Pharmacogenetics of adverse drug reactions. Adv. Pharmacol. 83, 155-190 (2018). 